\title{
Future Mobile Communication Services on Balance between Desire for Freedom and Trust
}

\author{
Yoshitoshi Murata \\ Faculty of Software and Information Science, Iwate Prefectural University, \\ E-mail: y-murata@iwate-pu.ac.jp
}

Received 9 March 2016;

Accepted 2 June 2016

\begin{abstract}
Some applications introduced in each $5 \mathrm{G}$ mobile communication project (5G-P) assume special use cases such as when many people simultaneously use their mobile phone in a restricted area like a stadium or when using traffic safety systems are in common. And, some of them such as mobile healthcare and the Internet of things (IOT) have already been presented by many people. However, the reasons those applications will be widely used are uncertain. And then, most authors of articles related to future mobile communication made no mention of business schemes such as who deploys network infrastructure or who provides service content.

However, histories of existing mobile communication markets may show applications that are different from the ones written in the above articles and new business schemes that are different from existing ones.

In this paper, I introduce results of analysing histories of existing mobile communication markets show that communication applications are always dominant and they have evolved based on users' desire for communication. The other hand, some SNSs who are recent communication application having privacy security problems have left from the mobile communication market. And then, results of analysing the business models of the network carriers show that deregulation of mobile communication carrier operators has expanded the
\end{abstract}

Journal of ICT, Vol. 4_l, 19-34.

doi: $10.13052 /$ jicts2245-800X.412

(c) 2016 River Publishers. All rights reserved. 
mobile communication market. On the other hand, I point out that excessive deregulation can cause problems, mainly security problems, for customers.

Keywords: mobile communication market, mobile evolution, 5G, contextaware communications, user-centric networking.

\section{Introduction}

Several projects aimed at developing 5G mobile communication systems are underway in various parts of the world. 5G-PPP [1] and METIS [2, 3] in the EU and the 5G Forum [4] in Korea have already issued white papers. 5GMF $[5,6]$ in Japan has signed a memorandum of understanding with 5G-PPP. Most such projects are based on special use cases in the 5G mobile (5GM) era and address the performance requirements for those cases. Moreover, they focus on the technologies needed and the challenges that must be overcome to meet those requirements. The use cases include smart cars, traffic collision avoidance, and heavy communication traffic volumes in a small area such as a stadium.

Some use cases introduced in 5G-PPP, 5GMF, and ETSI are used in common, and most applications addressed in the 5G Forum have already been presented by many people. However, the reasons those applications were expected to be widely used are unclear. Furthermore, most authors of articles related to future mobile communication failed to mention the enabling business schemes such as who will deploy the network infrastructure or who will provide service content even though the schemes dictate the structures and functions of $5 \mathrm{G}$ systems. In addition, the applications that have actually emerged in the mobile communications market differ from those mentioned in the published articles, and new business schemes differ from existing ones.

Most major mobile communication applications were developed in the latter half of the mobile communication period, so a new generation system was required to handle the growing required performance or number of subscribers. For example, the i-mode mobile Internet service in Japan was developed in the $2 \mathrm{G}$ era, but the performance of $2 \mathrm{G}$ mobile (2GM) was insufficient for full Internet service-the performance of 3G mobile (3GM) was needed. It is still unclear which existing applications will need 5GM performance.

In this paper, I analyse the dominant non-voice applications on current and previous mobile communication systems including paging services; and identify the primary factors that made them dominant. I also introduce analysis 
results showing that communication services are always dominant and that they have evolved in accordance with users' desire for communication. The other hand, some SNSs who are recent communication application having privacy security problems have left from the mobile communication market. In addition, I introduce the results of analysing network carrier business models showing that deregulation of mobile communication carrier operators has expanded the mobile communication market. On the other hand, I point out that excessive deregulation can cause problems, mainly security problems, for customers.

After introducing related work in Section 2, I analyse current and previous mobile communication applications and business models in Section 3 to clarify the primary factors that made them dominant. On the basis of these primary factors, I forecast in Section 4 which mobile communication applications and business schemes will be dominant. I conclude in Section 5 with a summary of the key points and a personal note.

\section{Related Works}

I introduce use cases and performance for each $5 \mathrm{G}$ project. Since applications that will be widely used in the future must have already appeared in the world, I introduce the new trends in mobile communication technologies.

\subsection{G Projects}

The $5 \mathrm{G}$ projects mentioned above sometimes compete and sometimes cooperate in taking the initiative on standardization and markets for 5GM. Their special use cases and applications target 2017-2020. They are listed in Table 1, along with their primary requirements.

Most of the use cases and applications must be limited for usage. Some cases would be very rare, and others could be solved by implementing suitable application software. For example, about ten years ago in Japan, so many young people visiting a shrine or temple on the first day of the new year were simultaneously sending messages with New Year greetings that the network carriers had to control the traffic volume to keep their systems operating. This led to an increased usage of social networking applications (SNSs) such as Facebook and LINE, which solved the New Year's Day traffic jam problem. This means that a huge amount of traffic in a stadium would not be a problem solved by $5 \mathrm{GM}$. The traffic efficiency and safety use case of METIS and the smart cars use case of the 5G Forum require super low latency. Moreover, 
Table 1 Use cases, applications, and requirements of some $5 \mathrm{G}$ projects

\begin{tabular}{|c|c|c|c|}
\hline & 5G PPP/5GMF & METIS & 5G Forum \\
\hline $\begin{array}{l}\text { Use cases and/or } \\
\text { applications }\end{array}$ & Stadium traffic & $\begin{array}{l}\text { Stadium traffic; } \\
\text { Traffic efficiency } \\
\text { and safety }\end{array}$ & $\begin{array}{l}\text { Smart cars; } \\
\text { Medical } \\
\text { education; } \\
\text { Games; } \\
\text { Disaster relief }\end{array}$ \\
\hline Peak data rate & $10 \mathrm{~Gb} / \mathrm{s}$ & $10 \mathrm{~Gb} / \mathrm{s}$ & $50 \mathrm{~Gb} / \mathrm{s}$ \\
\hline Data volume & $10 \mathrm{~Tb} / \mathrm{s} / \mathrm{km}^{2}$ & $\begin{array}{l}9 \text { Gbytes/h in busy } \\
\text { periods; } \\
500 \mathrm{~GB} / \mathrm{mo} / \\
\text { subscriber }\end{array}$ & - \\
\hline Number of devices & $1 \mathrm{M} / \mathrm{km}^{2}$ & $300 \mathrm{~K} /$ access point & - \\
\hline End-to-end latency & $5 \mathrm{~ms}$ & $5 \mathrm{~ms}$ & $1 \mathrm{~ms}$ \\
\hline
\end{tabular}

they also need the ability to identify vehicles and obstacles (including people) and to determine their locations. These latter two functions will be difficult to achieve. In addition, it is unclear whether these use cases and applications will be common in the $5 \mathrm{GM}$ era.

\subsection{Context-Aware Communications and Networking}

One of new trends in communication research is context-aware communications and networking (CACN). Most existing communication systems do not take into account the context of such entities as people, vehicles, phones, and base stations. In contrast, in CACN, the contexts of entities (both real and virtual) are taken into account in end-to-end communication [7]. The concept of CACN is very wide and is applied in all communications and networking layers, from the physical and networking layers to the transport and application layers. Misra et al. applied this concept to the location of nodes and the rate of dissemination in wireless sensor networks and thereby effectively reduced the end-to-end delay of disseminated data with different priorities in an energyefficient manner [8]. Researchers referred to the smart house, smart city, and so on would be included in CACN. Rao et al. proposed a context-event triggering mechanism in a smart cyber-physical space that works through energy harvesting [9]. Murata and Saito proposed a cloud service, called "cyber parallel traffic world" (CPTW), in which vehicles, pedestrians, and temporary obstacles exist and move in synchronization with their real-world counterparts [10]. In CPTW, drivers and pedestrians can communicate with other drivers and pedestrians by pointing to their positions rather than referring to an ID (e.g., a telephone number or address). 


\subsection{User-Centric Networking and Device-to-Device Communication}

Other new trends in communication research are user-centric networking (UCN) and device-to-device (D2D) communication. While 1 to $4 \mathrm{G}$ communication networks were deployed by network carriers, UCNs and D2D networks are created by the users themselves, who cooperate by sharing network applications and resources. They are thus characterized by spontaneous and grassroots deployment of wireless architectures [11]. In an article entitled "Spontaneous Smartphone Networks as a User-Centric Solution for the Future Internet," Aloi et al. describe building a local network using smartphones, WiFi access points (APs), Bluetooth devices, and network file systems that is organized in terms of spontaneous connectivity [12]. This approach could lead to low-cost operation, occasional super low latency, and high spectrum efficiency. However, if a malicious device terminal is implemented in a UCN as a relay node, that network would be risky to use. Important information could be stolen from a user's terminal. Therefore, security and trust functions are necessary for UCNs. Frangoudis and Polyzos identified the challenges of user centricity in terms of their effect on wireless networking architectures, particularly security, in an article entitled "Security and Performance Challenges for User-Centric Wireless Networking" [13].

\section{History of Evolution in Mobile Communication}

In biology, the evolution basically depends on the genetic mutation and the natural selection. The genetic mutation is caused by not only natural mutation but also viruses, radiation and so on [14]. Anyhow, whether new species of organisms can survive and thrive is dependent on coincidence. It is very difficult to predict which new species of organisms will thrive.

The other hand, in the mobile communication market, whether new applications, business models and so on are accepted by a lot of subscribers is mainly dependent on subscriber's values or preferences and social rules. Subscriber's values or preferences must not change so rapidly. This means that there would be solutions for which applications and so on will thrive in a history of mobile communication market. I analyse existing and past mobile communication applications and business models to clarify the primary factors that produce the dominant applications and business schemes. 


\subsection{Applications}

In most articles related to the evolution or future of mobile communication, $1 \mathrm{G}$ cellular phone systems, which provided only analog voice service, are considered to be the first mobile communication system. However, the paging system was actually the first mobile communication system for non-voice service. Prior to the introduction of $2 \mathrm{GM}$ phone systems, paging systems provided one-way messaging service in its half period. Users could send a message by using a push-button phone, a PC mail system such as Lotus Notes or Internet mail. Paging applications were mainly used by business people in the U.S. and the EU, and by young people, especially female high school students, in Japan.

The advent of $2 \mathrm{GM}$ led to the integration of $1 \mathrm{G}$ mobile (1GM) phone systems and paging systems (2GP), enabling two-way messaging service (Internet mail) and limited World Wide Web (WWW) service in Japan, as shown in Figure 1. In the EU and U.S., the two-way short messaging service (SMS) became widely used as a consumer communication service. Full WWW service using smartphones started in the latter half of the 2GM era, and becomes popular in the $3 \mathrm{GM}$ era. One of most popular application in $3 \mathrm{GM}$ era is the SNSs such as Facebook and LINE. They suit for a mobile communication service in fact, most mobile mail user shifts to SNS's messaging applications. Anyway, messaging applications are dominant in any mobile communication systems. The reason would be that since human are afraid to be isolated, they want someone to listen to what they say and get sympathy.

Researchers in both industry and academia university continue to develop new mobile communication systems, and network carriers are deploying them to enable subscribers to use their applications anytime and anywhere.

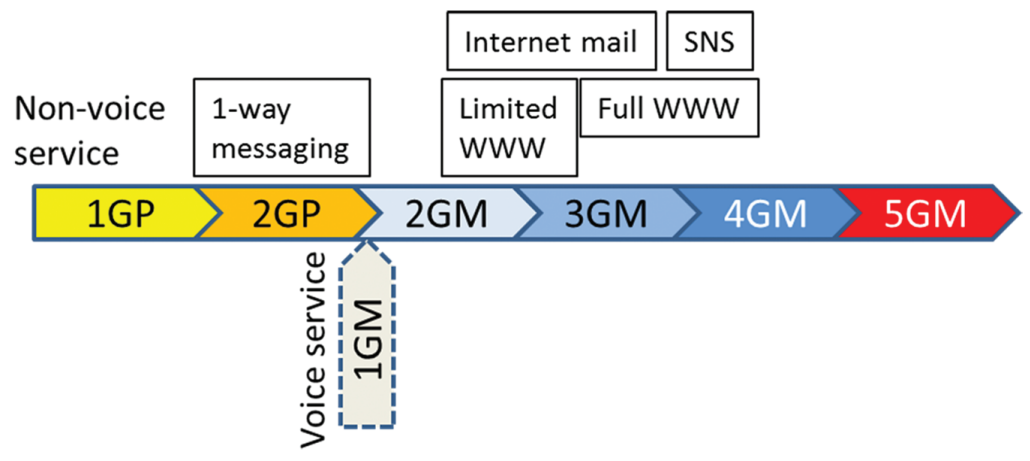

Figure 1 Evolution of non-voice applications in Japan. 
Users can connect their smartphones to the Internet through not only 3GM and 4GM but also WiFi. Generally, users can connect to the Internet at lower cost and with a higher data rate by using WiFi rather than 3GM or 4GM. When connecting through a carrier network, the user generally does not need to worry about security. However, when connecting through a WiFi access point, the user has to consider whether it is safe or not, because some of WiFi access points would be malicious [15]. Unfortunately, most users do not do this.

The evolution of messaging service from the one-way messaging to twoway messaging to SNS messaging has been accelerated by seeking freedom to easily communicate someone and to get sympathy. However, in the period introducing unguaranteed network resources, keeping trust for participants is very important to expand the mobile communication market.

\subsection{Business Schemes}

In most countries, a specified organization such as AT\&T, and NTT provided mobile communication service in the first era of mobile communication. The mobile market was closed, and there was no competition. Since then, mobile communication markets have been opened somewhat in most countries. The number of carriers is still limited, and operations are controlled by the government. This means that competition is restricted.

The standard mobile communication business model is a vertically integrated one. I proposed the open heterogeneous mobile network (OHMN) model to accelerate competition in the mobile communication market at the first Kaleidoscope in 2008 [16]. In this model, mobile communication business activities are horizontally arranged on five layers as shown in Figure 2.

Each layer corresponds to a core activity;

- Terminal layer: manufacturing and selling terminals.

- Network layer: deploying and providing access networks.

- Connection service layer: setting up voice communication path between end terminals through different access networks. (Mobile virtual network operators are on this layer)

- Platform layer: user authentication and charging.

- Contents \& application layer: developing and providing contents and applications for mobile users.

In the standard business model (1.0), the market is closed while in the proposed business model (2.0) the market is open. While carriers continue have the right to provide wired and wireless networks, connection applications, and 


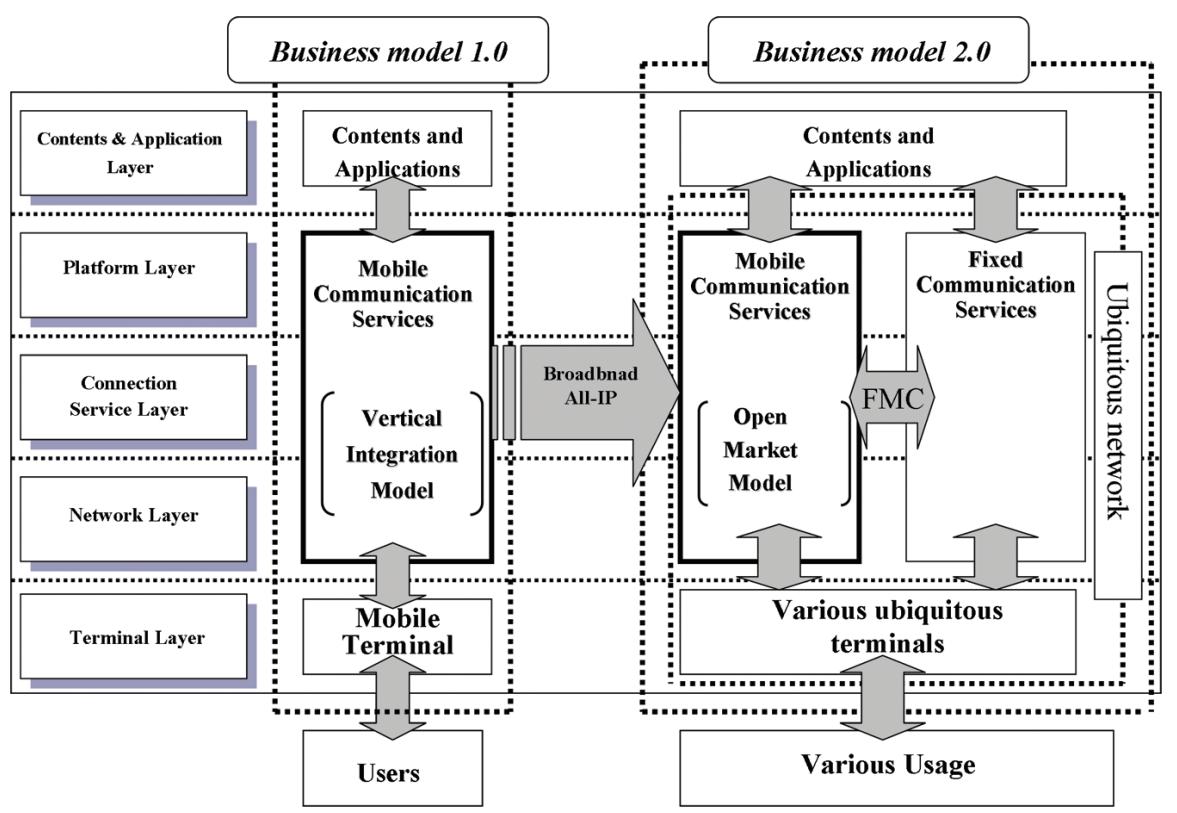

Figure 2 Open heterogeneous mobile network (OHMN) business layer model.

billing applications, newcomers such as Skype and LINE now provide a voice exchange application, that is the connection service, over the Internet, and most billing applications for contents and applications have shifted from carriers to credit service providers. And then, MVNOs provide a voice exchange service and an Internet connection service with rented wireless networks. These mean that most business layers except the wireless network layer are entirely open. There would be many people who want to deploy and provide wireless networks.

\section{Future Mobile Communication Service}

In this section, I forecast trends in service provision and business schemes.

\subsection{Service Provision}

I first analyze service provision from the standpoint of user freedom regarding "anytime, anywhere, anyone, and anything." It is very difficult to completely achieve "anytime, anywhere and anything" freedom, and the 5G projects present very special use cased which need advanced technologies to achieve 
them. However, there are some problems which do not need advanced technologies. The $5 \mathrm{G}$ projects make no mention of them. For example, mobile subscribers have to pay high roaming charges when they are out of their home country. Frequent visitors to foreign countries often buy a pre-paid subscriber identity module (SIM) card for each country to enable use of a smartphone or tablet without having to pay roaming charges in each country. Less frequent visitors typically do not use the mobile communication system but use WiFi instead. A roaming charge is simply an unfavorable aspect of mobile communication. It is not a technical problem to be solved. Since the principle involved is the same as the "increase of entropy principle," some network carriers could abolish the charge while others could continue to impose it.

Systems that use users' IDs to connect them do not provide "anyone" freedom. For example, a driver (A) stuck in a traffic jam cannot connect to a driver (B) at the front of the jam, since A does not know B's ID. This is one factor driving CACN research. It also led to my proposal to use the CPTW cloud service for solving traffic-related problems. Drivers using this service could learn the locations of other vehicles, pedestrians, and obstacles being tracked by the service and could communicate with each other by pointing to an object such as an automobile or pedestrian.

The concept of CACN is a very broad; the use cases described above are only a few of its potential applications. I call the actions in the above use cases "context-aware messaging (CAM)". A context and ID exchange (CIDX) server is needed for CAM, as shown in Figure 3 Its database is used to relate a subscriber's profile (including ID) to his or her context. When a person accesses the CIDX server, a graphical user interface such as a map on which context objects are plotted is displayed. The CPTW described in Section 2.2 is

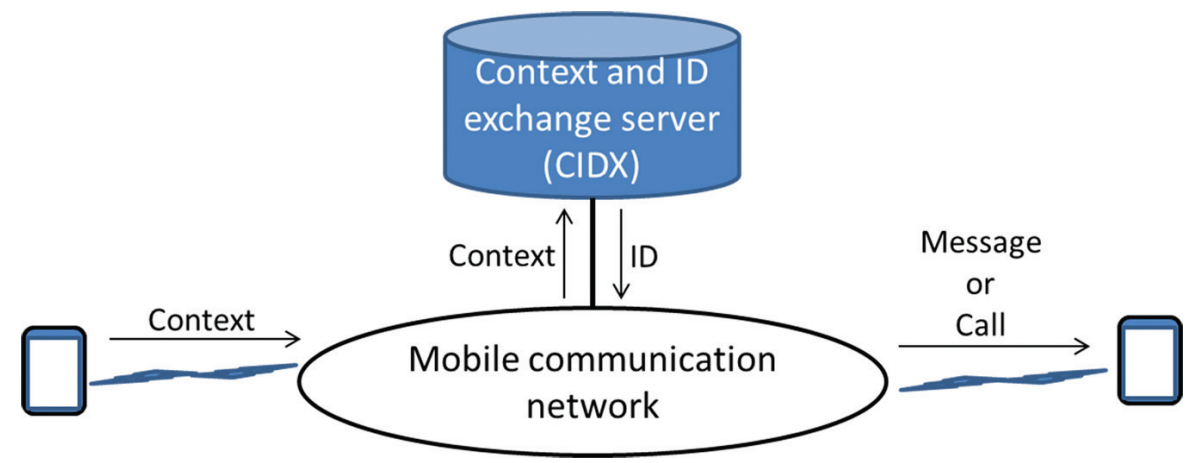

Figure 3 CAM system structure. 
also one of CIDX servers. For example, if Mr. A, who has heart disease, falls down, his smartphone automatically sends an emergency signal including its location information. After receiving the signal, the CIDX server searches the context database for the IDs of doctors or other persons near Mr. A who have experience in the use of an automated external defibrillator (AED) and sends an appropriate person a message with a description of the problem and the location information.

While CAM would be very useful for law-abiding people, as shown by the example above, it would also be very useful for criminals. For example, a criminal could send a forged context signal that summons the police, enabling the criminal to easily commit a crime elsewhere. Therefore, the application of CAM to applications requires that trust between participants be maintained.

I next forecast the trend of post-SNS services. There are currently two types of SNS: one type is messaging in which the destinations are anonymous, such as in Twitter; the other is messaging in which the destinations are limited to a group, such as in Facebook. Both two-way and flexible group messaging capabilities are provided by existing messaging services such as e-mail and SNS. Most SNSs support attaching files and sharing feelings ("like", etc.). This situation seems to be that there is no room for the SNS developing. However, current messaging services have critical problems. The father of modern corporate management, Peter Drucker, said some very important things about communication [17];

i. The most important thing in communication is hearing what isn't said.

ii. Commutation is expectation - meaning recipients will only hear or see whatever they expect to be able to hear or see.

iii. Communication requires shared experience. There can be no communication if it is conceived as going from the 'I' to the 'Thou'. Communication works only from one member of 'us' to another. Communication in an organization is not a means of organization. It is the mode of organization.

I use these insights in my forecast of the direction for next-generation messaging services.

First, let's consider the first two insights. In the case of face to face (F2F) communication, it is possible to obtain information from facial and voice expressions in addition to what is said. This is impossible to do in message communication. A message written expressing goodwill is sometimes perceived as expressing bad-will. This is attributed to the second insight and to the absence of facial and voice expressions. Therefore, some messaging services such as LINE provide emoji for facial expressions and thereby convey a feeling. In the case of LINE, creating and selling emoji has become 
a business. Unfortunately, a set of facial expression emoji has not been standardized. Moreover, there are cultural differences in facial expressions. I expect that one of the next-generation messaging services will support facial expression emoji that are transparent between cultures.

The third insight means that sharing experiences activates communication between members in a group. And members in a group sharing experiences would feel a strong affinity for the other members. I forecast that at least one post-SNS messaging service will be one in which experiences are shared. For example, a subscriber could send messages to others who are in the same location, such as in a restaurant, to share their experience such as tasting good food or enjoying the atmosphere. Subscribers could exchange messages with other subscribers in the same shopping mall to obtain useful information. Climbers could exchange messages with other climbers on the same mountain to get updates on weather conditions and so on. This type of service is a Twitterlike application in which destinations are not anonymous but limited by the context. This idea of context-limited messaging has already been implemented for some network games, such as Ingress, in which one group of players competes against another group. The members in a group cooperate and share experiences with each other during a game by messaging. Trust within a group is very important for sharing experiences since subscribers are sometimes geographically very close. If a subversive person joined a group, the group's performance could be undermined.

\subsection{Business Schemes for Service Providers}

As I wrote in Section 3.2, only the wireless network has not been entirely liberalized. The reason is that the frequency assignment to carrier operators is managed by governments. In this section, I consider possible business schemes in entirely liberalized wireless networks.

\subsubsection{Network layer}

As discussed in Sections 2.3 and 3.2, some parts of wireless networks must be open in the future. Before discussing the business scheme for the network layer, we have to consider what types of wireless units that will be available. Following three types of wireless units must be provided by manufactures in the future as same as $4 \mathrm{GM}$;

- WU-1: XGM wireless unit; for outdoor use.

- WU-2: XGM wireless unit like a femtocell in 3GM and 4GM; for indoor use.

- WiFi-AP: WiFi access point; mainly for indoor use. 
Here, the acronym "XGM" presents the future generation mobile system. WU1 units must be provided to carrier operators, the same as for existing wireless units; only carrier operators will deploy them. WiFi-AP units will be provided as an open terminal, the same as existing WiFi-AP units, so anyone can buy one.

Opening up the WU-2 units the same as the WiFi-AP units would activate the market. If anyone can buy WU-2 units, WU-2 and WiFi-AP units could connect to the carrier's exchange units or a third party's exchange units through the Internet, as shown in Figure 4. Each exchange unit has a subscriber identification function and call exchange function. When a subscriber's terminal connects to a WU-2 or WiFi-AP unit, the unit accesses a contracted exchange unit to identify the terminal's ID. In case of voice communication, the exchange unit creates a session path between the terminal and itself and keeps it while the two parties are talking.

Scenarios for WiFi-Ap or WU-2 connecting to which exchange unit would depend on three primary factors;

F1: WU-2 is allowed to use the frequency band that has been allocated to WiFi.

F2: Frequency bands for XGM are independently allocated to each specified carrier or shared among carriers.

F3: Which takes the initiative, the carrier operator or the third party?

I first discuss an exchange unit for WiFi-AP. An operation of such a unit depends on F3, i.e., who has the initiative, as shown in Figure 5. There are two alternatives for the unit.

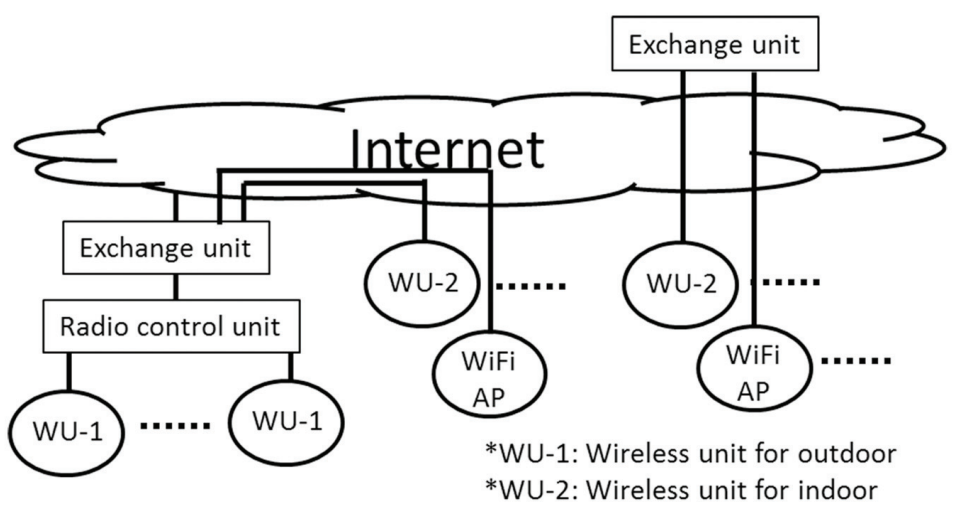

Figure 4 Structure of future mobile communication system. 


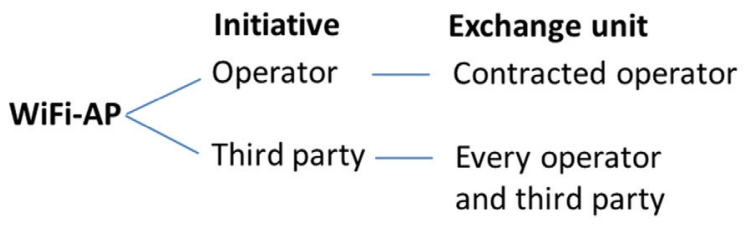

Figure 5 Scenarios of connecting exchange unit for WiFi-AP.

In contrast, the operation of an exchange unit for WU-2 depends on every factor. Therefore, its operation is rather complex, as shown in Figure 6. Scenario 8 (S8) is the one that will most activate the mobile communication market. I am looking forward to the government allowing WU-2 units to use the WiFi band and to standardization for XGM systems sharing the same frequency band. Two other problems related to opening the network layer are:

- Maintaining security while freely selling WU-2 and WiFi-AP units.

- Sustaining the business of third parties who deploy WU-2 or WiFi-AP units for general subscribers.

At least, WU-2 or WiFi-AP units have to be provided the same as the mobile terminal. The ordinance of radio equipment for mobile terminals should be applied to WU-2 or WiFi-AP units. When they will be connected to an operator's exchange unit, a chip like a SIM chip has to be mounted in the unit. If possible, an exchange unit should be able to examine whether a WU-2 or WiFi-AP unit has been contaminated with malicious programs such as computer viruses.

Alternatives for sustaining a third party's business are basically billing for usage and sending advertisements. They depend on the third party involved.

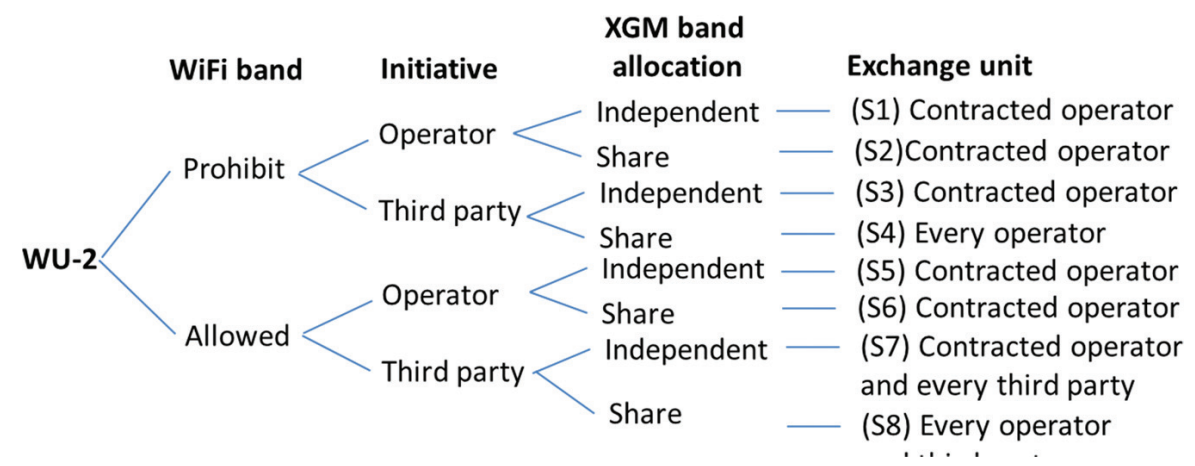

Figure 6 Scenarios of connecting exchange unit for WiFi-AP. 
A shopping mall, for example, could send information customized for each subscriber and shop. A restaurant could use a WU-2 or WiFi-AP unit to take orders or attract customers. In the "taking orders" example, the unit would need a function for charging for the provider's service. In the "attracting customers" example, the unit would need a function for using cloud applications.

\subsubsection{Connection layer}

As mentioned above, voice exchange applications are already being provided by several cloud service providers such as Skype and LINE. Skype can already handle exchanges between carriers for voice communication. However, they cannot handle fast hand-off service between WiFi routers. Technically, the IEEE has already standardized fast-hand-off for WiFi as IEEE 802.11r or 11ai [18]. The fast hand-off service between WiFi-AP and/or WU-2 units would be provided by some providers.

\subsubsection{Platform layer}

Billing for smartphone applications already has been opened to outsiders, and many such applications are in the Google Play Store and the Apple Store. It is possible to buy them by credit cards and prepaid cards. IC/Mobile wallet applications are now widely used in several countries such as Japan. It is possible to pay by IC card or mobile phone. Apple provides the Apple-pay app for its iPhone.

The Internet of Things (IOT) will be ubiquitous in the near future. For example, the fee for parking in most parking lots in Japan is paid using an on-site charging system, which is not cost effective. The application of IC/Mobile wallet or Apple-pay applications to them would be more convenient and effective. In the future, such charging systems will utilize the IOT and make our lives easier.

\section{Conclusion}

The analysis I have presented of existing mobile communication applications clarified the primary factors that made such applications dominant. I hypothesized that the evolution of mobile communication service was accelerated on the basis of a balance between participants' desire for the freedom and suspicion. My forecast of mobile communication applications on the basis of these factors shows that sharing experiences will be the next stage in the evolution of messaging applications. The spread of very low power wireless stations using the WiFi band and of standardized XGM systems sharing the 
same frequency band will activate the mobile communication market. Finally, fast hand-off applications between WiFi-access points and very low power wireless stations, and billing applications to utilize the Internet of Things will be introduced in a future.

These discussed developments should result in improved human ability, more convenient living, and new markets.

\section{References}

[1] 5G PPP. The 5G Infrastructure Public Private Partnership. Available at: http://5g-ppp.eu/

[2] Mobile and wireless communications Enablers for the Twenty-twenty Information Society (METIS). Available at: https://www.metis2020.com/

[3] Osseiran, A., et al. (2014). "Scenarios for 5G Mobile and Wireless Communications: The Vision of the METIS Project," IEEE, Commun. Mag. 52, 26-35.

[4] ICT-317669 METIS Project. (2013). "Scenarios, Requirements and KPIs for $5 G$ Mobile and Wireless System,” Del. D1.1. Available at: https://www.metis2020.com/documents/deliverables/

[5] Park, Y. (2014). $5 G$ Vision and Requirements of 5 G Forum, Korea. Available at: https://www.itu.int/dms_pub/itu-r/oth/0a/06/R0A0600005F0001 PDFE.pdf

[6] 5GMF. The Fifth Generation Mobile Communications Promotion Forum. Available at: http://5gmf.jp/en/

[7] Wu, J., et al. (2014). "CONTEXT-AWARE NETWORKING AND COMMUNICATIONS: PART 1," IEEE Commun. Mag. 14-15.

[8] Misra, S., Das, S. N. and Obaidat, M. (2014). "Context-Aware Quality of Applications in Wireless Sensor Networks," IEEE Commun. Mag. 16-23.

[9] Rao, V. S., Nambi, S. N. A. U., Prasad, R. V., and Niemegeers, I. (2014). "On Systems Generating Context Triggers through Energy Harvesting," IEEE Commun. Mag. 70-77.

[10] Murata, Y. and Saito, S. (2014). "Cyber Parallel Traffic World' Cloud Service in 5G Mobile Networks," J. ICT 2, 65-86.

[11] Sofia, R. et al. (2014). "USER-CENTRIC NETWORKING AND APPLICATIONS: PART 2," IEEE Commun. Mag. 16.

[12] Aloi, G. et al. (2014). "Spontaneous Smartphone Networks as a UserCentric Solution for the Future Internet," IEEE Commun. Mag. 26-33. 
[13] Frangoudis, A. and Polyzos, G. C. (2014). "Security and Performance Challenges for User-Centric Wireless Networking," IEEE Commun. Mag. 48-55.

[14] Ryan, F. (2009). Virolution, FPR-Books Ltd, HarperCollins. WiFi ALLIANCE, Security. Available at: http://www.wi-fi.org/discoverwi-fi/security

[15] Murata, Y., Murakami, H., Hasegawa, M. (2008). "Architecture and Business Model of Open Heterogeneous Mobile Network", ITU-T, Kaleidoscope.

[16] Drucker, P. (1999). The Essential Drucker, HarperCollins e-books; Reissue.

[17] 802.11r. (2008). IEEE Standard for Information technology - Local and metropolitan area networks - Specific requirements - Part 11: Wireless LAN Medium Access Control (MAC) and Physical Layer (PHY) Specifications Amendment 2: Fast Basic Applicationset (BSS) Transition.

\section{Biography}

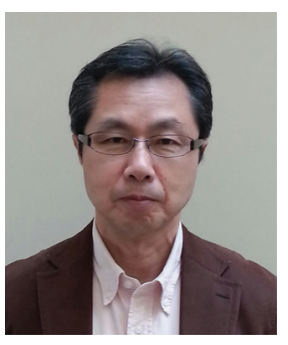

Y. Murata received his M.E from Nagoya University, Japan. He received his Ph.D from Shizuoka University, Japan. From 1979 to 2006, he was belonging to NTT and NTT DoCoMo and developing mobile communication systems, mobile terminals and services. Since 2006, he is a professor of faculty of Software and Information Science, Iwate Prefectural University. Prof. Murata was awarded the best paper of the ITU-T "Innovations in NGN" Kaleidoscope Academic Conference 2008. His research interests include mobile communication, medical information and ITS. He is a member of IEEE, IEICE and IPSJ. 